Assume $p \in V\left(P^{*}\right)$ is a solution to $\bar{S}$. If $\omega$ is a vector space basis of $K^{*} / K$ then each $D \in B^{*}$ can be written in the form $D=\sum d^{(j)} \omega^{(j)}$, $d^{(j)} \in K\{y\}$. Since $\omega$ is also a vector space basis of $M^{*} / M, d^{(j)}(\alpha)=0$ and $d^{(j)} \in B$. Therefore each $I \in J^{*}$ can be written in the form $I=\sum I^{(j)} \omega^{(j)}, I^{(j)} \in J$. Since $p$ is a solution to $S, p$ is a solution to $J$. Therefore, $p$ is a solution to $J^{*}$ and hence to $\bar{S}^{*}$. Thus $p \in H$ and $H$ is the set of solutions to $\bar{S}$ in $V\left(P^{*}\right)$.

The hypotheses of the Lemma are satisfied and we obtain $\operatorname{dim} H$ $=\operatorname{dim}(H \cap V(C))=\operatorname{dim} G$. Further, since $P^{*}$ is algebraically closed, $\operatorname{dim} H=\operatorname{dim} \bar{S}^{*}$. Since $c$ is a generic zero of $\bar{S}$, and $P^{*}$ is an algebraic extension of $C$, t.d. $(M, K)=\operatorname{dim} \bar{S}=$ t.d. $(C(c), C)=$ t.d. $\left(P^{*}(c), P^{*}\right)$ $=\operatorname{dim} \bar{S}^{*}$. Therefore $\operatorname{dim} G=$ t.d. $(M, K)$, and the proof is complete.

\title{
REFERENCES
}

1. R. Cohn, Difference algebra, Wiley, New York, 1965.

2. M. Epstein, On the theory of Picard-Vessiot extensions, Ann. of Math. 62 (1955), 528-547.

3. C. Franke, Picard-Vessiot theory of linear homogeneous difference equations, Trans. Amer. Math. Soc. 3 (1963), 491-515.

4. - Solvability of linear homogeneous difference equations by elementary operations, Proc. Amer. Math. Soc. 1 (1966), 240-246.

5. - Reducible linear homogeneous difference equations, (to appear).

6. I. Kaplanksy, An introduction to differential algebra, Hermann, Paris, 1957.

Seton Hall University

\section{A NOTE ON ABSOLUTELY FREE ALGEBRAS}

\section{K.-H. DIENER AND G. GRÄTZER ${ }^{1}$}

1. Introduction. A free algebra $\mathfrak{B}^{(\mathfrak{m})}(\tau)$ over the class $K(\tau)$ of all (universal) algebras of a fixed type $\tau$ is called an absolutely free algebra. The following is a simple characterization of absolutely free algebras.

ThEOREM. Let $\mathfrak{A}$ be an algebra of type $\tau$, and let $M$ be a generating set of $\mathfrak{A}$. Then the following two conditions are equivalent:

(i) $\mathfrak{A}$ is absolutely freely generated by $M$;

(ii) $M$ is independent in every extension $\mathfrak{B}$ of $\mathfrak{A}$ with rank $\mathfrak{B} \leqq|M|$ and if there are nullary operations, then $\mathfrak{A}^{c} \cong \mathfrak{P}^{(0)}(\tau)$.

Received by the editors May 10, 1966.

1 Research supported by the National Science Foundation under grant number GP-4221. 
REMARK 1. Independence is meant in the sense of [2]. The rank of $\mathfrak{B}$ is the smallest cardinal $\mathfrak{n}$, such that $\mathfrak{B}$ has a generating set of cardinality $\mathfrak{n}$. Finally, $\mathfrak{A}^{c}$ denotes the subalgebra of $\mathfrak{A}$ generated by the set $A_{0}$ of all values of nullary operations.

REMARK 2. This theorem was announced by the first author at the Meeting of the International Mathematical Union, Stockholm, 1962, see also [1]. The present proof is a simplification of the original one.

REMARK 3. In condition (ii) "rank $\mathfrak{B} \leqq|M|$ " can be replaced by "rank $\mathfrak{B}=|M|$."

\section{Proof of the Theorem.}

(i) implies (ii). Let (i) hold. Set $|M|=\mathfrak{m}$. Then $\mathfrak{A} \cong \mathfrak{P}^{(\mathfrak{m})}(\tau)$, so the first part of (ii) is obvious. If there are constant operations, then $\mathfrak{A}^{c} \cong\left(\mathfrak{P}^{(\mathfrak{m})}(\tau)\right)^{c} \cong \mathfrak{P}^{(0)}(\tau)$, hence (ii) holds.

(ii) implies (i). Let $\mathfrak{A}=\langle A ; F\rangle$ satisfy (ii), $M=\left\{m_{i} \mid i \in I\right\},|I|$ $=\mathfrak{m}, \mathfrak{B}^{(\mathfrak{m})}(\tau)=\left\langle P^{(\mathfrak{m})}(\tau) ; F\right\rangle$ and (if there are nullary operations) let $\phi$ be the isomorphism of $\mathfrak{A}^{c}$ with $\mathfrak{P}^{(0)}(\tau)$. Let $\left\{b_{i} \mid i \in I\right\}$ be a basis of $\mathfrak{P}^{(\mathfrak{m})}(\tau)$ (the basis of $\mathfrak{B}^{(\mathfrak{m})}(\tau)$ is unique, however we will not make use of this fact). We can assume that $A$ and $P^{(\mathfrak{m})}(\tau)$ are disjoint and both are disjoint to a set $\left\{n_{i} \mid i \in I\right\}=N$.

If $\tau$ is the void type (there are no operations) or every operation is nullary, then the statement is trivial. We shall now distinguish two cases.

Case 1. There is at most one operation $f$ which is not nullary, and $f$ is unary. Take $B=N \cup A$; retain the definition of the nullary operations and of $f$ on $A$ and put $f\left(n_{i}\right)=m_{i}$, for $i \in I$. This defines on $B$ an algebra $\mathfrak{B}$. Then rank $\mathfrak{B}=\mathfrak{m}$, thus by (ii), the mapping $m_{i} \rightarrow n_{i}(i \in I)$ can be extended to a homomorphism $\psi$ of $\mathfrak{A}$ into $\mathfrak{B}$ (in fact, on to $\mathfrak{B}$ ). We claim that this implies that $\mathfrak{A}$ is absolutely freely generated by $M$. Indeed, denoting by $f^{k}$ the $k$ th iterate of $f$, we have to check that $(\alpha) \mathfrak{I}^{c} \cong \mathfrak{B}^{(0)}(\tau) ;(\beta) f^{k}(x)=f^{l}(y)$ implies $k=l$ and $x=y$, where $x, y$ $\in M \cup A_{0}$. Condition $(\alpha)$ holds by assumption. Condition $(\beta)$ follows from $(\alpha)$ if $x, y \in A_{0}$; let $x \in A_{0}, y \notin A_{0}$ (or the symmetric case), say $y=m_{i}$. Then $f^{k}(x)=f^{l}\left(m_{i}\right)$ implies that $f^{l}\left(m_{i}\right) \in A^{c}$; choose $l$ to be minimal with $b=f^{l}\left(m_{i}\right) \in A^{c}$. Then $b \psi=b=f^{l}\left(m_{i}\right) \psi=f^{l}\left(m_{i} \psi\right)=f^{l}\left(n_{i}\right)$ $\in A^{c}$, which is a contradiction, since if $l=0$, we get $n_{i} \in A^{c}$, and if $l \geqq 1$, we get $f^{l-1} f\left(n_{i}\right)=f^{l-1}\left(m_{i}\right) \in A^{c}$. Finally, let $x, y \in M$, say $x=m_{i}, y=m_{i}$. We prove $(\beta)$ by induction on $k$. For any $k, l, f^{k}\left(m_{j}\right)$ $=f^{l}\left(m_{i}\right)$ implies $f^{k}\left(m_{j}\right) \psi=f^{l}\left(m_{i}\right) \psi$, hence $f^{k}\left(n_{j}\right)=f^{l}\left(n_{i}\right)$. If $k=0$ (or $l=0$ ), then by $N \cap A=\phi, l=0$ (resp. $k=0$ ) and $n_{j}=n_{i}$, i.e. $m_{j}=m_{i}$. If $k>0$, then also $l>0$, therefore $f^{k}\left(n_{j}\right)=f^{l}\left(n_{i}\right)$ implies $f^{k-1}\left(m_{j}\right)$ $=f^{l-1}\left(m_{i}\right)$. Thus, by the induction hypothesis, $k-1=l-1$, i.e. $k=l$, and $m_{j}=m_{i}$. 
It is fairly easy to see that $(\alpha)$ and $(\beta)$ imply the generalized Peano axioms (for the notion of a generalized Peano algebra see [1]). Thus $\mathfrak{A}$ is absolutely freely generated by $M$.

REMARK ADDED IN PROOF (May 15, 1967). The following example shows that the condition " $\mathfrak{A} \cong \mathfrak{P}^{(0)}(\tau)$ " cannot be omitted from condition (ii) of the Theorem. Set $A=N \cup N / \sim$, where $N$ is the set of natural numbers, $\sim$ is "congruence modulo $n$ ", $n \in N, n>1$. Consider the algebra $\mathfrak{A}=\langle A ; f, g\rangle$, where $f$ is a unary operation, defined to be the successor operation on $N$ and the successor operation modulo $n$ on $N / \sim ; g$ is a nullary operation whose value is $0 / \sim$. Then $M=\{0\}$ is independent in every extension of $\mathfrak{A}$, but $M$ is not absolutely independent.

However, if we assume that there are nullary operations then the first part of (ii) can be weakened to " $M$ is independent in $\mathfrak{A}$ "; this will be proved in a forthcoming paper of the first author.

Case 2. Either (a) there exists an $n$-ary operation $f$ with $n>1$, or (b) there exist two unary operations $g_{0}$ and $g_{1}$.

Set $B^{\prime}=A \cup P^{(\mathfrak{m})}(\tau) \cup N$; we get the set $B$ from $B^{\prime}$ by identifying every $a \in A^{c}$ with $a \phi \in P^{(0)}(\tau) \subseteq P^{(\mathfrak{m})}(\tau)$. Define the operations on $B$ as follows: we retain the definition of the operations on $A$ and $P^{(\mathfrak{m})}(\tau)$; we fix an $a \in A$; in case (a), we set $f\left(n_{i}, a, a, \cdots, a\right)=m_{i}$, and $f\left(a, n_{i}, a, \cdots, a\right)=b_{i}$, for $i \in I$; in case (b), we set $g_{0}\left(n_{i}\right)=m_{i}$ and $g_{1}\left(n_{i}\right)=b_{i}$, for $i \in I$. All the undefined operations and as yet undefined values equal $a$.

For the resulting algebra $\mathfrak{B}$, rank $\mathfrak{B}=\mathfrak{m}$ is obvious, since every generating system has to contain $N$ and $N$ generates $\mathscr{B}$.

$\mathfrak{A}$ is a subalgebra of $\mathfrak{B}$, rank $\mathfrak{B}=\mathfrak{m}$, so by (ii), $M$ is independent in $\mathfrak{B}$. In particular, the mapping $m_{\boldsymbol{i}} \rightarrow b_{i}$, for $i \in I$, can be extended to a homomorphism $\psi$ of $\mathfrak{A}$ in to $\mathfrak{B}$. The image of $\mathfrak{A}$ under $\psi$ is $\mathfrak{P}^{(\mathfrak{m})}(\tau)$; thus $\psi$ is a homomorphism of $\mathfrak{A}$ onto $\mathfrak{P}^{(\mathfrak{m})}(\tau)$, with $m_{i} \psi=b_{i}$, for $i \in I$. Since $\mathfrak{P}^{(\mathfrak{m})}(\tau)$ is free over $K(\tau)$, the mapping $b_{i} \rightarrow m_{i}$, for $i \in I$, can be extended to a homomorphism $\chi$ of $\mathfrak{B}^{(\mathfrak{m})}(\tau)$ into $\mathfrak{A}$. But $m_{\boldsymbol{i}}(\psi \chi)=m_{\boldsymbol{i}}$, for $i \in I$, hence $\psi \chi$ is the identity mapping on $A$ and so $\psi$ is an isomorphism of $\mathfrak{A}$ and $\mathfrak{B}^{(\mathfrak{m})}(\tau)$, which concludes the proof.

\section{REFERENCES}

1. K.-H. Diener, Zur Theorie der absolut freien und verwandter Algebren, Ph.D. Thesis, Univ. of Cologne, 1963.

2. E. Marczewski, $A$ general scheme of the notions of independence in mathematics, Bull. Acad. Polon. Sci. Sér. Sci. Math. Astronom. Phys. 6 (1958), 731-736.

University OF COLOGNE AND

Pennsylvania State University 